\title{
BMJ Open Development and evaluation of an algorithm-based tool for Medication Management in nursing homes: the AMBER study protocol
}

\author{
Susanne Erzkamp, ${ }^{1}$ Olaf Rose ${ }^{1,2}$
}

To cite: Erzkamp S, Rose 0. Development and evaluation of an algorithm-based tool for Medication Management in nursing homes: the AMBER study protocol. BMJ Open 2018;8:e019398. doi:10.1136/ bmjopen-2017-019398

- Prepublication history and additional material for this paper are available online. To view these files, please visit the journal online (http://dx.doi. org/10.1136/bmjopen-2017$019398)$.

Received 6 September 2017 Revised 29 January 2018 Accepted 20 March 2018

Check for updates

${ }^{1}$ Elefanten-Apotheke, gegr. 1575, Steinfurt, Germany

${ }^{2}$ Department of

Pharmacotherapy and

Translational Research,

University of Florida College of Pharmacy, Gainesville, Florida, USA

Correspondence to

Susanne Erzkamp;

amber-study@gmx.de

\section{ABSTRACT}

Background Residents of nursing homes are susceptible to risks from medication. Medication Reviews (MR) can increase clinical outcomes and the quality of medication therapy. Limited resources and barriers between healthcare practitioners are potential obstructions to performing MR in nursing homes. Focusing on frequent and relevant problems can support pharmacists in the provision of pharmaceutical care services. This study aims to develop and evaluate an algorithm-based tool that facilitates the provision of Medication Management in clinical practice.

Methods and analysis This study is subdivided into three phases. In phase I, semistructured interviews with healthcare practitioners and patients will be performed, and a mixed methods approach will be chosen. Qualitative content analysis and the rating of the aspects concerning the frequency and relevance of problems in the medication process in nursing homes will be performed. In phase II, a systematic review of the current literature on problems and interventions will be conducted. The findings will be narratively presented. The results of both phases will be combined to develop an algorithm for MRs. For further refinement of the aspects detected, a Delphi survey will be conducted. In conclusion, a tool for clinical practice will be created. In phase III, the tool will be tested on MRs in nursing homes. In addition, effectiveness, acceptance, feasibility and reproducibility will be assessed. The primary outcome of phase III will be the reduction of drug-related problems (DRPs), which will be detected using the tool. The secondary outcomes will be the proportion of DRPs, the acceptance of pharmaceutical recommendations and the expenditure of time using the tool and inter-rater reliability.

Ethics and dissemination This study intervention is approved by the local Ethics Committee. The findings of the study will be presented at national and international scientific conferences and will be published in peerreviewed journals.

Trial registration number DRKS00010995.

\section{INTRODUCTION}

Pharmaceutical Care is defined as the "pharmacist's contribution to the care of individuals in order to optimise medicines use and improve health outcomes'. ${ }^{1}$ Medication

\section{Strengths and limitations of this study}

The process to develop an algorithm for Medication Management in nursing homes uses a variety of consecutive methods.

- The resulting tool is tested on several aspects, such as effectiveness, feasibility and acceptance, by multidisciplinary healthcare providers.

- The inclusion of clinical practitioners at the development of the tool is believed to support translation into standard care.

- The algorithm needs to balance limited time and resources in community care to detect as many relevant drug-related problems as possible.

- The limited duration and size of this uncontrolled study requires further research and testing.

Review (MR) and Medication Management are current instruments of Pharmaceutical Care that have proven to be effective in reducing drug-related problems (DRPs), increasing the quality of the medication regimen and improving medical outcomes in various settings and indications. ${ }^{2-9} \mathrm{MR}$ is defined by the Pharmaceutical Care Network Europe (PCNE) as 'a structured evaluation of a patient's medicines with the aim of optimising medicines use and improving health outcomes. This entails detecting drug related problems and recommending interventions'. ${ }^{10}$ Medication Management involves 'patient-centred care to optimise safe, effective and appropriate drug therapy. Care is provided through collaboration with patients and their healthcare teams'. ${ }^{11}$ Pharmaceutical care interventions are especially meaningful in high-risk populations. ${ }^{12}$ Residents of nursing home facilities are a highly vulnerable patient group whose medication deserves special attention. In addition to geriatric age and dependence on care, multimorbidity and polymedication are frequently related to this patient population. ${ }^{13}{ }^{14}$ Inappropriate 
medication is related to a poor quality of life, high morbidity, preventable adverse drug events, increased risk for falls, repeated hospitalisations and manifold physician contacts. ${ }^{15-18}$ Several approaches to optimise the quality of the medication regimen have been tested. MR, multidisciplinary case conferences, education and coaching are examples of pharmaceutical interventions that have been studied successfully in nursing homes, ${ }^{19-23}$ and DRPs could be reduced and the quality of medication could be enhanced. The effects of pharmaceutical care on further clinical outcomes, such as mortality or quality of life, are uncertain. ${ }^{19}{ }^{22}$ This might be due to the limited size and length of most pharmaceutical studies. However, structured and collaborative Medication Management seems to be particularly supportive in this setting but is rarely implemented into standard care in Germany. ${ }^{524}$ Barriers of implementation might include time, resources and compensation. Limited experience in performing MR and Medication Management and assessing entry barriers in nursing home facilities might be an additional reason for withholding these services to the residents. Structure and guidance have been identified as tools to support pharmacists in the administration of MR. ${ }^{25}$ Setting higher standards of medication quality in nursing homes might be even more challenging as further action to encounter the patients, nurses and physicians is required. Tailored screening tools and standardised communication forms are helpful to guide and support pharmacists in performing MR and have been developed for various scenarios. ${ }^{26-28}$ For pharmacists with limited experience, the TIMER tool has shown to be effective in performing MR to a certain extent but has not been updated or refined any more. ${ }^{29}$ A contemporary tool that is tailored to the demands of pharmacists willing to conduct Medication Management in a nursing home could be helpful in overcoming existing barriers of implementation. However, it needs to take the previously mentioned aspects of multidisciplinary collaboration, structured guidance and limited time and resources into account.

\section{Aims and objectives}

The aim of the AMBER (Development and evaluation of an algorithm in Medication Management for best practice. Effectiveness of the intervention and translation into standard care for nursing home residents) study is to develop and test an algorithm-based tool that supports pharmacists in performing structured collaborative Medication Management in an appropriate time frame. The tool should take frequent and relevant problems in the medication process of residents of nursing homes into account and needs to consider the special circumstances of this setting. In addition to demonstrating effectiveness in detecting and solving DRPs, which are defined as 'events or circumstances involving drug therapy that actually or potentially interfere with desired health outcomes,' the tool needs to be highly feasible for community pharmacists. ${ }^{30}$ To assure a high patient benefit, multidisciplinary approaches need to be facilitated by the tool. In addition, it should be developed with the utmost available evidence and serve the patient.

\section{METHODS AND ANALYSIS}

This study is registered at the German Clinical Trials Register (registration number DRKS00010995). It is funded by Apothekerstiftung Westfalen-Lippe (non-commercial foundation). The funding source has no role in the design of this study and will not have any role in its execution, the analysis or interpretation of the data or the decision to submit results. SE is the guarantor for this paper and the study. All of the authors drafted the protocol.

\section{Overview of the study}

This study will be performed in three phases (figure 1). Phase I consists of interviews with healthcare practitioners and patients. In phase II, a systematic review will be performed, and the results will be combined with the outcomes of phase I to form an algorithm. Following a Delphi approach, the determined aspects of the algorithm will be presented to an expert panel to refine the algorithm. In phase III, the algorithm will be tested in patients.

\section{Phase I: practitioner and patient interviews Purpose}

Semistructured interviews with physicians, pharmacists, nurses and patients will be performed to identify frequent and relevant aspects of the medication process in nursing homes. The involvement of different healthcare practitioners was chosen to consider the different perspectives. Furthermore, this approach should assure practical relevance and feasibility and support the pragmatic attitude of the study. Patient interviews will be conducted to consider the goals of patients. The results of phase I will be to consider creation of the algorithm.

\section{Methods}

Phase I of the study is based on a mixed methods approach that includes qualitative and quantitative aspects. Physicians, pharmacists, nurses and patients will be interviewed about their experience, requirements and expectations regarding problems, risks and goals at the medication process in nursing homes. We will strive for five to ten experts in each group. Semistructured interviews with healthcare practitioners and patients will be conducted.

Physicians, pharmacists and nurses at phase I are required to have more than 1 year's experience in nursing home care before the interview and are required to work in a facility in North Rhine-Westphalia. Participation is voluntary. Patients at the panel will be residents of a nursing home facility and will be suggested by the particular head of nursing service. In addition, they must be able to understand and answer the questions without assistance. Open-ended questions covering uncertainties 
Phase I

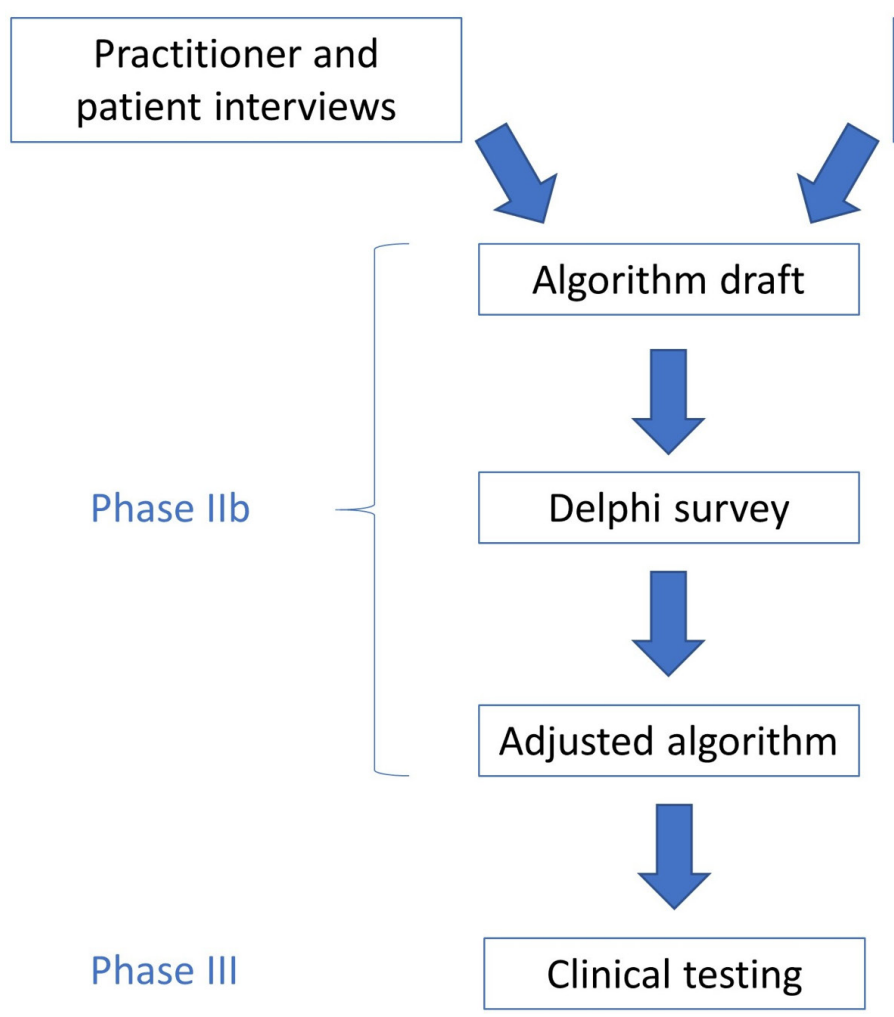

Phase Ila

Figure 1 Study flow.

and problems in the medication process will be asked. A qualitative content analysis described previously by Mayring ${ }^{31}$ will be performed with the software MAXQDA 12. The frequencies of coded categories are analysed. Furthermore, 51 specific aspects of therapy and DRPs covering general challenges, patient goals, communication barriers, medical goals and pharmaceutical aspects will be assessed. The 51 aspects were elaborated together with practitioners of each profession in a first approach. Additional aspects can be added by physicians, pharmacists, nurses and patients. Each aspect will be rated separately for frequency and relevance on a scale from 1 to 5 (with 1 being infrequent or irrelevant to 5 being frequent and relevant). Patients will be asked to rate a limited questionnaire of 24 aspects for relevance. The restrictions are created to reduce patient burden and based on the appraisal of two nurses. The frequency of the aspects is deleted in the patient version of the questionnaire, as they do not have an overview regarding the prevalence in other patients. Parameters rated by more than $50 \%$ of the participants with an average score of 3 or higher for frequency and relevance will be chosen as meaningful. Subsequently, the top scores per group will be considered for the algorithm.

The planned time frame for phase I is 6 months (July 2016-December 2016).

\section{Phase lla: literature review}

A literature review will be performed by following the Medical Research Council guidance. ${ }^{32}$ The protocol was prepared according to Preferred Reporting Items for Systematic review and Meta-Analysis Protocols (PRISMA-P) $2015,{ }^{33} 34$ and the review is registered in PROSPERO (registration number CRD42017065002). The PRISMA-P 2015 checklist is presented in online supplementary appendix 1 .

The objective of phase IIa is to systematically review the literature for relevant aspects of MR in nursing homes. The interventions will be analysed, and general challenges, patient goals, communication barriers, medical goals and pharmaceutical aspects will be considered. The intended review aims to answer the question of which problems arise most frequently and which aspects are most relevant. It includes three consecutive steps. In step 1 , a review of the existing reviews will be done. In step 2, interventional studies that have been published after the last review will be searched and analysed. In step 3, studies on frequent problems in a nursing home setting will be examined, especially those that have not been covered by steps 1 and 2 .

\section{Step 1: review of reviews \\ Purpose}

To develop an intervention, the use of the best available evidence is recommended. ${ }^{32}$ Reviews on interventions to optimise medication therapy in nursing homes should provide an indication of effective interventions or parts of them. This step aims to answer the questions of which interventions have already been developed, how effective they are and which aspects of the medication therapy can be improved by a MR. 


\section{Methods}

Systematic reviews, reviews and meta-analyses will be included. Participants must be nursing home residents (65 years and older). Studies with a geriatric population living outside nursing home facilities will be excluded, except studies that investigated both groups and separately provide data.

Any intervention that could be part of a MR will be considered.

Included studies can either be controlled or uncontrolled trials with standard care as a potential comparator. The endpoints of interest are hospitalisation, mortality and falls, among others. In cases where the outcomes will be DRPs or potential inadequate medication, they must be reported in detail.

The studies will need to be finished, and the results will need to be published. Articles in English and German will be included.

The following electronic bibliographic databases will be searched:

- MEDLINE/PCM (via PubMed)

- PsycINFO (via EBSCOhost)

- Cochrane Database of Systematic Reviews (via Cochrane Library)

- CINAHL (via EBSCOhost)

- International Pharmaceutical Abstracts (via EBSCOhost)

- NHSEED/DARE (NHS Economic Evaluation Database/Database of Abstracts of Reviews of Effects) (via CRD, Centre for Reviews and Dissemination)

Additionally, the reference lists of the included studies and reviews will be hand-searched.

Articles published between 1 January 2000 and 31 March 2017 will be conducted. This restriction should ensure the current nature of the data and consider the progress of healthcare. An example of a search strategy is presented in online supplementary appendix 2. Citavi 5 software will be used for data management and to remove duplications. Additional duplications will be removed by hand. One review author (SE) will conduct the search in the databases and will extract the titles and abstracts for analyses. Two reviewers (SE and OR) will independently screen the publications for inclusion. Discussion and consensus will resolve potential disagreements.

A data extraction form that will be used to collect data from eligible studies will be developed for each step. Data extraction will be conducted by one reviewer (SE) with verification by another (OR). Disagreements will be resolved by discussion and consensus. At least the following data will be extracted: the type of intervention, the tools used, conditions and outcomes. Outcomes will be dependent on the endpoints of the regarded studies, for example, the influence on mortality, hospitalisation rates, falls, quality of life and time spent for care. Studies will be checked with the AMSTAR checklist. ${ }^{35}$ Studies with poor quality will not be removed but the methods will be reported as far as possible.
Step 2: review of recent studies

Purpose

At step 2, interventional studies for optimising medication therapy that will be published after the reviews from step 1 will be included; hence; these studies could not have been considered by these earlier reviews.

\section{Methods}

The methods are similar to step 1 . The interventional studies will be examined following the template for intervention description and replication (TIDieR) checklist. ${ }^{36}$ Studies with poor quality will not be removed, but the methods and the description of the intervention will be reported as far as possible.

\section{Step 3: review of common problems \\ Purpose}

In addition to the results of the reviews and interventional studies, frequent problems can also be described by non-interventional studies. The focus in this step is on reported problems and issues in the medication process in nursing homes that are independently detected from an intervention. We aim to supplement problems that might not be the subject of the interventional studies.

\section{Methods}

Specific studies that are not covered by steps 1 and 2 will be examined, for example, observational studies, qualitative studies and guidelines (the additional procedure is similar to steps 1 and 2).

\section{Data synthesis}

A narrative synthesis will be provided. This is an approach 'that relies primarily on the use of words and text to summarise and explain the findings of the synthesis'. ${ }^{37}$ Descriptions of effective interventions and frequent problems will be summarised. Because of the potential inhomogeneity of the outcomes, a meta-analysis is expected to be inappropriate.

\section{Phase Ilb: creating the algorithm and a Delphi survey}

The results of phase I will be compared with the results of phase IIa. The determined aspects will be checked for eligibility and feasibility in the algorithm. Every aspect will be approved by the authors and considered for inclusion. A summarisation will be conducted, and the aspects will be evaluated in a Delphi survey with 10 or more experts using the software SurveyMonkey with a fivepoint Likert scale. The remarks of the expert panel will be incorporated into the algorithm. The experts are pharmacists and experienced researchers in the field of MR, especially in the nursing home setting. They will be asked for agreement or disagreement regarding the proposed aspects. Consensus will be defined as an agreement of $70 \%$ or higher and a median higher than 3 .

The planned time frame for phase II is 6 months (January 2017-July 2017). 


\section{Phase III: clinical testing}

The study protocol follows the Standard Protocol Items: Recommendations for Interventional Trials (SPIRIT) 2013 statement, ${ }^{38} 39$ and the study design is developed in line with the manual for the design of non-drug trials in primary care by Joos et al. ${ }^{40}$ The SPIRIT 2013 checklist and the WHO Trial Registration Data Set are presented in online supplementary appendices 3 and 4 . Conducting the TIDieR checklist to describe the intervention will be conducted later when reporting the outcomes. ${ }^{36}$

\section{Purpose}

In phase III, the developed algorithm will be tested for effectiveness by performing a MR in nursing home patients. Acceptance of the pharmaceutical recommendations will be measured using a feedback form. In addition, the feasibility of the algorithm will be investigated. Several pharmacists will use and rate the feasibility after conducting MR on written case scenarios.

\section{Methods}

A single-armed prospective study design will be used. Reductions in the detected DRPs will be the indicator for effectiveness of the intervention.

The planned time frame for phase III is 12 months (July 2017-June 2018).

\section{Study setting}

This study will be conducted in nursing homes in North Rhine-Westphalia, Germany.

\section{Eligibility criteria}

The following inclusion criteria will be applied:

- Patients aged $\geq 65$ years

- Residency in a nursing home facility

- Multimorbidity: at least two chronic diseases (25)

- Polymedication: at least five chronic systemic medications

- Signed informed consent (if necessary through a legally authorised representative)

Exclusion criterion:

- Participation in other clinical studies at the current time

A withdrawal of consent will be possible at any time and will lead to the discontinuation of the intervention. Data on dropouts will not be included in the analyses of the primary outcome. The patients will retain all rights regarding their personal data and its deletion.

\section{Intervention}

After recruitment, a one-time MR will be performed by the project manager using the developed algorithm $\left(\mathrm{t}_{0}\right)$. The patient data will be collected from nursing homes and physicians. Information about the actual condition of the patient can be supplemented by the patients themselves or by the nurses at the facilities. The results of the MR will be communicated to nurses and physicians using SOAP (subjective data, objective data, assessment and plan) notes and will be discussed if required. ${ }^{41}$
SOAP notes will cover detected problems in medication therapy and recommendations to solve them. Acceptance will be measured using a feedback form filled out by physicians and nurses and depending on the recommendations. At a 3-month postintervention follow-up $\left(t_{1}\right)$, the medication of the patient will be evaluated for changes and the status of the detected DRPs will be reviewed. The number of solved and unsolved DRPs will be collected.

In addition to this main analysis, an advanced MR will be performed for 10 patients as a benchmark. This type of review is based on medication history, patient information and clinical information according to the PCNE definition. ${ }^{42}$ In contrast to the PCNE definition, patient information can also be derived from nurses in this particular setting. The results of the advanced MR will be compared with the results of the MR performed using the algorithm. The number and type of detected DRPs will be compared and analysed by the project manager. The differences will be descriptively presented.

Feasibility and reproducibility of the algorithm will be tested by five or more pharmacists using 10 similar patient cases. The case description and patient information will be handed out to the pharmacists anonymously in a written form and the experiences will be descriptively presented. The timeline of phase III is shown in figure 2.

Standard care will be provided during the trial and interventions will be supplementary. This study will monitor for the potential harm of the intervention by recording adverse drug reactions following the implemented suggestions.

\section{Outcomes}

The primary outcome will be the change in the number of DRPs classified according to 'The PCNE Classification V 8.01 ' and detected using the algorithm. ${ }^{30}$ Potential DRPs will be limited to the aspects covered by the algorithm.

Secondary outcomes

- DRPs classified according to PCNE V.8.01 (number and type)

- Acceptance measurement of physicians and nurses using an acceptance form and classified according to PCNE V.8.01

- Number of DRPs classified according to PCNE V.8.01 and detected by an advanced MR

- Reproducibility of the analysis and feasibility of the algorithm (time spent and inter-rater reliability)

\section{Sample size}

Regarding the assumption of a mean reduction of one DRP per patient with a SD of 2, a calculation will be performed by the institute for biometric and clinical research of the Westfälische Wilhelms-University Münster (Institut für Biometrie und Klinische Forschung, IBKF). The null hypothesis, whether the number of DRPs does not differ, will be tested with a two-sided Wilcoxon signedrank test with a significance level of 0.05 . The estimated sample size to improve a power of $80 \%$ will be 75 patients. 
Recruitment and signed informed consent

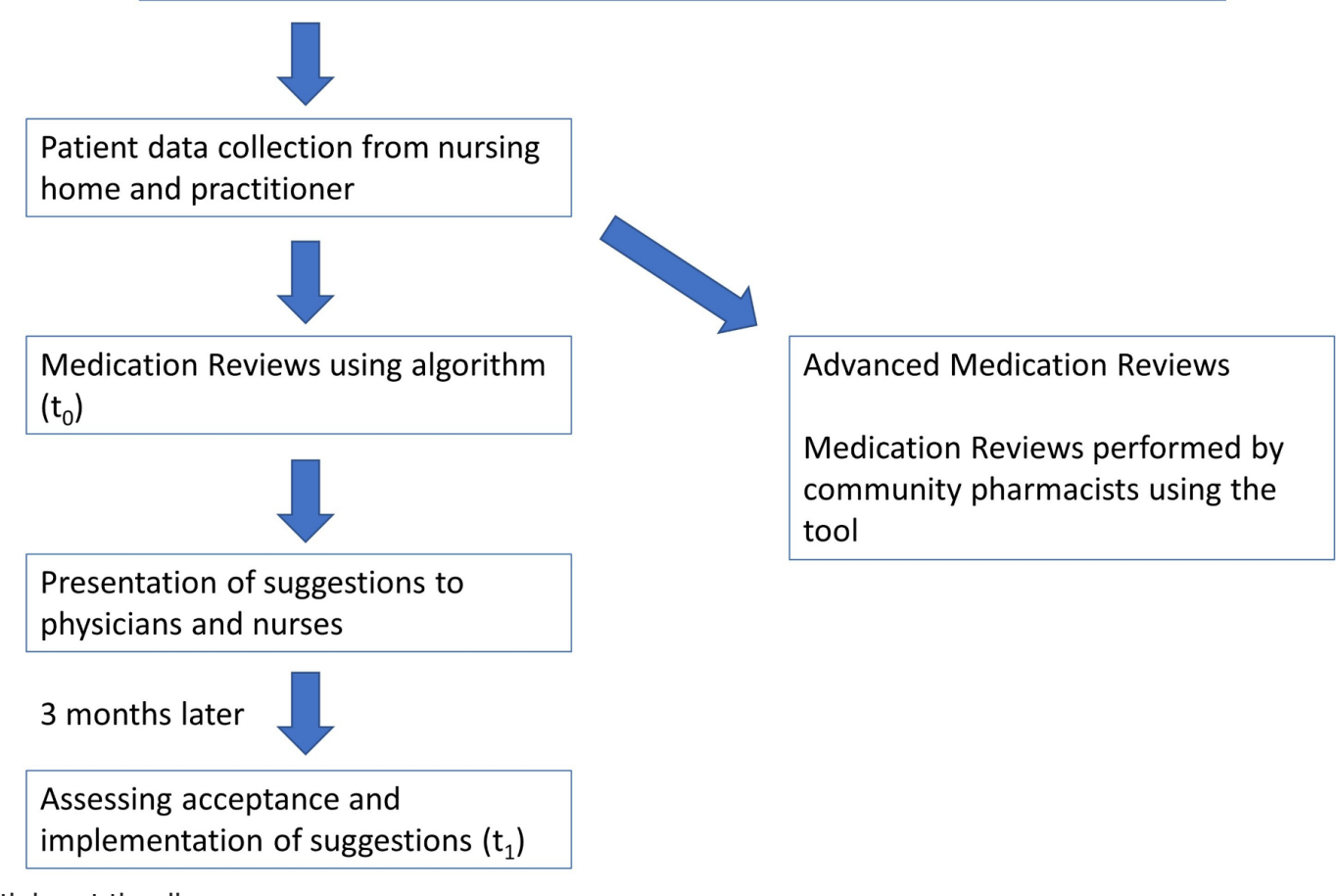

Figure 2 Participant timeline.

Considering dropouts, a sample size of 100 patients is intended.

\section{Recruitment}

As a first step, three nursing homes in North Rhine-Westphalia will be asked to participate in the study. All of the patients of the participating nursing homes will be screened for inclusion and exclusion criteria and subsequently asked to join the study.

In case of an insufficient number of participating patients, additional nursing homes will be approached.

\section{Data collection}

During the study, the following data will be collected and documented using Microsoft Excel software:

- Name and study number

- Age and sex of participant

- Medication, including active pharmaceutical ingredients, dosage and pharmaceutical form

- Morbidities, laboratory parameters and vital signs

These data will be collected from nursing homes' and physicians' documentation.

Furthermore, the results of the MR will be documented using password-protected Excel sheets. All of the reasons for withdrawal or dropout will be recorded in the study.

\section{Data management}

Patient-related data will be used for performing an MR using the algorithm and for communication with nurses and practitioners. For all of the statistical analyses and other MR, anonymised data will be used.

\section{Statistical methods}

Baseline and demographic characteristics will be descriptively analysed. The reductions in DRPs will be tested with a two-sided Wilcoxon signed-rank test with a significance level of 0.05 .

\section{ETHICS AND DISSEMINATION}

The study intervention is approved and will be conducted based on the principles of the Declaration of Helsinki. If amendments of the protocol are necessary, the date of each amendment, a description of the change and the rational will be given. Changes will not be incorporated into the protocol. The project manager will obtain informed consent from potential trial participants or legally authorised representatives. Written information will be given to all of the study patients. Confidentiality will be guaranteed by anonymising patient data where applicable. Only the project manager will have complete data for communication with nurses and physicians. An analysis of the final data by IBKF and the project manager will be performed.

The goal of the study is the development of an algorithm as an underlying basis for use as a tool. The tool is intended to be applied into standard care and might be used by community pharmacies engaged in nursing home care. The findings of this study will be presented at national and international scientific conferences and will be published in peer-reviewed journals. There are no publication restrictions. 
Patients, nursing homes and physicians who participate in this study will be offered a final report on the study results.

\section{Methods against bias and for quality assurance}

To ensure the data quality and to avoid missing data or processes that are not adherent with the study protocol, the study sites will be visited for clinical monitoring (to determine whether the diagnoses, clinical data and medication were current). Furthermore, several routines will be established to prevent or detect incorrect and inconsistent data entry and incomplete data. Additionally, regular training sessions will be conducted.

\section{DISCUSSION}

The aim of this study is the development of an algorithm leading to the development of a tool to support community pharmacists in performing Medication Management in nursing homes. The tool is then tested for effectiveness, feasibility and practicability.

\section{Development}

The tool needs to detect as many relevant DRPs as possible but only take limited time and resources into account. Hence, both aspects need to be balanced. Each step of the algorithm needs to be based on evidence. Therefore, a mixed methods approach that incorporates interviews with multidisciplinary practitioners and patients, a systematic review, a Delphi survey and testing and refinement of the underlying algorithm is chosen. This approach in developing the algorithm is quite comprehensive compared with other tools as it needs to cover both explicit criteria, which 'can be applied with little or no clinical judgement,' and implicit criteria, which also take a patient's preferences into account. ${ }^{43}$ This is in contrast to more confined medication-safety tools, which may depend on a mere Delphi survey. ${ }^{43} 44$ The complex method in developing the AMBER tool is believed to provide a higher probability of included items than a limited approach. For example, taking practitioners' experience into account helps to rate current trends in misprescribing, as these may vary from time to time. ${ }^{45}$ As shown by da Costa et al, tools to evaluate medication regimens differ in the number of detected DRPs, whereas it is unclear if a higher number leads to a greater patient benefit or vice versa for over-reporting. ${ }^{26}$ In this study, the tool is developed solely for the medication of nursing home residents and needs to cover the specific requirements of this setting.

\section{Testing}

The developed tool is aimed to test for effectiveness in nursing homes in Germany in 100 patients. The number of relevant tool-detected DRPs is compared with the results of an advanced MR. Feasibility is evaluated by providing community pharmacists with tool and patient cases. The results of the pharmacists' test cases are compared with the study team's results. A survey on the feasibility and practicability will be conducted, and the time to perform a MR using the tool will be developed.

\section{Multidisciplinary approach}

Medication Management implicates further and multidisciplinary activities after a Medication Review is performed. At a nursing home, cooperation with nurses and physicians is vital to determine an effect on patients' medication, even though attitudes on patient-oriented approaches might vary. ${ }^{46-48}$ In regard to these considerations, the tool needs to lead pharmacists toward multidisciplinary cooperation. Nurse and physician acceptance is measured using a standardised feedback form and based on the PCNE classification of DRPs. ${ }^{30}$

\section{Strengths and limitations}

A potential strength of the study might be the variety of methods, the high numbers of underlying data and the large base of studies included in the development of the algorithm. Inclusion of clinical practitioners at the development of the tool is believed to support translation into standard care.

This study faces several limitations. Confined resources do not allow a larger and controlled study. Even though a complex method is used in developing the tool, it can be only a compromise to detect and solve a sufficient number of relevant DRPs. A comprehensive approach surely could perform better but has limited feasibility on translation into the aspired community setting. The short duration of the study intervention and the restricted study collection might not disclose all aspects as it is powered only for the primary endpoint. Other aspects might show up as relevant during the intervention phase.

Acknowledgements The authors acknowledge the contribution and support of Dr. Juliane Köberlein-Neu, Laura Kerschke, MSc and Dr. Udo Puteanus.

Contributors Conception and design: SE, OR. Administrative support: SE. Provision of study materials or patients: SE, OR. Collection and assembly of data: SE. Data analysis and interpretation: SE, OR. Manuscript writing: SE, OR. Final approval of manuscript: SE, OR.

Funding This study is funded by Apothekerstiftung Westfalen-Lippe (non-commercial foundation).

Competing interests SE received a speaker honorarium by MSD. OR received speaker honoraria from Bayer, Boehringer Ingelheim, Lilly, Medac, MSD, Novartis and Omnicell. The authors declare that there are no conflicts of interest regarding the publication of this article.

Patient consent Not required.

Ethics approval Ethics Committee of the Medical Association of Westphalia-Lippe and of the University of Münster, approval number 2017-350-f-S.

Provenance and peer review Not commissioned; externally peer reviewed.

Open Access This is an Open Access article distributed in accordance with the Creative Commons Attribution Non Commercial (CC BY-NC 4.0) license, which permits others to distribute, remix, adapt, build upon this work non-commercially, and license their derivative works on different terms, provided the original work is properly cited and the use is non-commercial. See: http://creativecommons.org/ licenses/by-nc/4.0/

(C) Article author(s) (or their employer(s) unless otherwise stated in the text of the article) 2018. All rights reserved. No commercial use is permitted unless otherwise expressly granted. 


\section{REFERENCES}

1. Allemann SS, van Mil JW, Botermann L, et al. Pharmaceutical care: the PCNE definition 2013. Int J Clin Pharm 2014;36:544-55.

2. Barnett MJ, Frank J, Wehring $\mathrm{H}$, et al. Analysis of pharmacistprovided medication therapy management (MTM) services in community pharmacies over 7 years. J Manag Care Pharm 2009;15:18-31.

3. Chisholm-Burns MA, Kim Lee J, Spivey CA, et al. US pharmacists effect as team members on patient care: systematic review and meta-analyses. Med Care 2010;48:923-33.

4. Lindenmeyer $\mathrm{A}$, Hearnshaw $\mathrm{H}$, Vermeire $\mathrm{E}$, et al. Interventions to improve adherence to medication in people with type 2 diabetes mellitus: a review of the literature on the role of pharmacists. $J$ Clin Pharm Ther 2006;31:409-19.

5. Stuijt CC, Franssen EJ, Egberts AC, et al. Appropriateness of prescribing among elderly patients in a Dutch residential home: observational study of outcomes after a pharmacist-led medication review. Drugs Aging 2008;25:947-54.

6. Viktil KK, Blix HS. The impact of clinical pharmacists on drug-related problems and clinical outcomes. Basic Clin Pharmacol Toxicol 2008;102:275-80.

7. Machado M, Bajcar J, Guzzo GC, et al. Sensitivity of patient outcomes to pharmacist interventions. Part II: Systematic review and meta-analysis in hypertension management. Ann Pharmacother 2007;41:1770-81.

8. Machado M, Nassor N, Bajcar JM, et al. Sensitivity of patient outcomes to pharmacist interventions. Part III: systematic review and meta-analysis in hyperlipidemia management. Ann Pharmacother 2008;42:1195-207.

9. Köberlein-Neu J, Mennemann H, Hamacher S, et al. Interprofessiona Medication management in patients with multiple morbidities. Dtsch Arztebl Int 2016;113:741-8.

10. Pharmaceutical Care Network Europe. Position Paper on the PCNE definition of Medication Review. 2016 http://www.pcne.org/workinggroups/1/medication-review (accessed 12 Jun 2017).

11. Canadian Pharmacists Association, Canadian Society of Hospital Pharmacists, Association of Faculties of Pharmacy of Canada and Institute for Safe Medication Practices Canada. Definition of Medication Management. https://www.pharmacists.ca/educationpractice-resources/professional-development/medicationmanagement/ (accessed 10 Jan 2018).

12. Rose $\mathrm{O}$, Mennemann $\mathrm{H}$, John $\mathrm{C}$, et al. Priority setting and influential factors on acceptance of pharmaceutical recommendations in collaborative medication reviews in an ambulatory care setting analysis of a cluster randomized controlled trial (WestGem-Study). PLoS One 2016;11:e0156304

13. Tamura BK, Bell CL, Inaba M, et al. Outcomes of polypharmacy in nursing home residents. Clin Geriatr Med 2012;28:217-36.

14. Patterson SM, Cadogan CA, Kerse N, et al. Interventions to improve the appropriate use of polypharmacy for older people. Cochrane Database Syst Rev 2014;10:CD008165

15. Hammond T, Wilson A. Polypharmacy and falls in the elderly: a literature review. Nurs Midwifery Stud 2013;2:171-5.

16. Endres HG, Kaufmann-Kolle P, Steeb V, et al. Association between potentially inappropriate medication (pim) use and risk of hospitalization in older adults: an observational study based on routine data comparing PIM Use with use of PIM alternatives. PLoS One 2016;11:e0146811.

17. Gallagher $P$, Barry $P, O^{\prime}$ Mahony $D$. Inappropriate prescribing in the elderly. J Clin Pharm Ther 2007;32:113-21.

18. Projovic I, Vukadinovic D, Milovanovic O, et al. Risk factors for potentially inappropriate prescribing to older patients in primary care. Eur J Clin Pharmacol 2016;72:93-107.

19. Alldred DP, Kennedy MC, Hughes C, et al. Interventions to optimise prescribing for older people in care homes. Cochrane Database Syst Rev 2016;2:CD009095.

20. Hughes CM, Lapane KL. Pharmacy interventions on prescribing in nursing homes: from evidence to practice. Ther Adv Drug Saf 2011;2:103-12

21. Forsetlund L, Eike MC, Gjerberg E, et al. Effect of interventions to reduce potentially inappropriate use of drugs in nursing homes: a systematic review of randomised controlled trials. BMC Geriatr 2011;11:16.

22. Wallerstedt SM, Kindblom JM, Nylén K, et al. Medication reviews for nursing home residents to reduce mortality and hospitalization: systematic review and meta-analysis. $\mathrm{Br} J \mathrm{Clin}$ Pharmacol 2014;78:488-97.

23. van Welie S, Wijma L, Beerden T, et al. Effect of warning symbols in combination with education on the frequency of erroneously crushing medication in nursing homes: an uncontrolled before and after study. BMJ Open 2016;6:e012286.

24. Erzkamp S, Rose O. Medikamentenmanagement oder Medikationsmanagement? Deutsche Apotheker Zeitung 2016;156:1616-21.

25. Niquille A, Lattmann C, Bugnon O. Medication reviews led by community pharmacists in Switzerland: a qualitative survey to evaluate barriers and facilitators. Pharm Pract (Granada) 2010;8:35-42.

26. da Costa FA, Periquito C, Carneiro MC, et al. Potentially inappropriate medications in a sample of Portuguese nursing home residents: Does the choice of screening tools matter? Int J Clin Pharm 2016;38:1103-11.

27. Bulloch MN, Olin JL. Instruments for evaluating medication use and prescribing in older adults. J Am Pharm Assoc (2003) 2014;54:530-7.

28. Marasinghe KM. Computerised clinical decision support systems to improve medication safety in long-term care homes: a systematic review. BMJ Open 2015;5:e006539.

29. Lee SS, Schwemm AK, Reist J, et al. Pharmacists' and pharmacy students' ability to identify drug-related problems using TIMER (Tool to Improve Medications in the Elderly via Review). Am J Pharm Educ 2009;73:52

30. Pharmaceutical Care Network Europe. Classification for Drug related problems version 8.01. http://www.pcne.org/upload/files/215_PCNE classification_V8-01.pdf (accessed 18 Aug 2016).

31. Mayring P. Qualitative inhaltsanalyse: grundlagen und techniken. 12th edn. Weinheim: Beltz, 2015.

32. Craig P, Dieppe P, Macintyre S, et al. Developing and evaluating complex interventions: the new Medical Research Council guidance. BMJ 2008;337:a1655.

33. Moher D, Shamseer L, Clarke M, et al. Preferred reporting items for systematic review and meta-analysis protocols (PRISMA-P) 2015 statement. Syst Rev 2015;4:1.

34. Shamseer L, Moher D, Clarke M, et al. Preferred reporting items for systematic review and meta-analysis protocols (PRISMA-P) 2015 elaboration and explanation. BMJ 2015;349:g7647.

35. Shea BJ, Grimshaw JM, Wells GA, et al. Development of AMSTAR: a measurement tool to assess the methodological quality of systematic reviews. BMC Med Res Methodol 2007;7:10.

36. Hoffmann TC, Glasziou PP, Boutron I, et al. Better reporting of interventions: template for intervention description and replication (TIDieR) checklist and guide. BMJ 2014;348:g1687.

37. Popay J, Roberts H, Sowden A, et al. Guidance on the conduct of narrative synthesis in systematic reviews: a product from the ESRC Methods Programme: Lancaster University, 2006.

38. Chan AW, Tetzlaff JM, Altman DG, et al. SPIRIT 2013 statement: defining standard protocol items for clinical trials. Ann Intern Med 2013;158:200-7.

39. Chan AW, Tetzlaff JM, Gøtzsche PC, et al. SPIRIT 2013 explanation and elaboration: guidance for protocols of clinical trials. $B M J$ 2013;346:e7586

40. Joos S, Bleidorn J, Haasenritter J, et al. [Manual for the design of non-drug trials in primary care, taking account of Good Clinical Practice (GCP) criteria]. Z Evid Fortbild Qual Gesundhwes 2013;107:87-92

41. American College of Clinical Pharmacy. Standards of practice for clinical pharmacists. Pharmacotherapy 2014;34:794-7.

42. Pharmaceutical Care Network Europe. PCNE statement on medication review. 2013 http://www.pcne.org/upload/files/150 20160504_PCNE_MedRevtypes.pdf (accessed 30 Sep 2016).

43. Page RL, Linnebur SA, Bryant LL, et al. Inappropriate prescribing in the hospitalized elderly patient: defining the problem, evaluation tools, and possible solutions. Clin Interv Aging 2010;5:75-87.

44. Holt S, SchmiedI S, Thürmann PA. Potentially inappropriate medications in the elderly: the PRISCUS list. Dtsch Arztebl Int 2010;107(31-32):543-51.

45. Halvorsen $\mathrm{KH}$, Selbaek G, Ruths S. Trends in potentially inappropriate medication prescribing to nursing home patients: comparison of three cross-sectional studies. Pharmacoepidemiol Drug Saf 2017;26:192-200.

46. Ruths S, Straand J, Nygaard HA. Multidisciplinary medication review in nursing home residents: what are the most significant drug-related problems? The Bergen District Nursing Home (BEDNURS) study. Qual Saf Health Care 2003:12:176-80.

47. Brulhart MI, Wermeille JP. Multidisciplinary medication review: evaluation of a pharmaceutical care model for nursing homes. Int $J$ Clin Pharm 2011;33:549-57.

48. Turner JP, Edwards S, Stanners M, et al. What factors are important for deprescribing in Australian long-term care facilities? Perspectives of residents and health professionals. BMJ Open 2016;6:e009781. 